\title{
Tighter constraints of multiqubit entanglement in terms of Rényi- $\alpha$ entropy
}

\author{
Meng-Li Guo ${ }^{1}, \mathrm{Bo}_{\mathrm{Li}}{ }^{2} *$ Zhi-Xi Wang ${ }^{3}$, and Shao-Ming Fei ${ }^{3,4}$ \\ ${ }^{1}$ Department of Mathematics, East China University of Technology, Nanchang 330013, China \\ ${ }^{2}$ School of Mathematics and Computer science, Shangrao Normal University, Shangrao 334001, China \\ ${ }^{3}$ School of Mathematical Sciences, Capital Normal University, Beijing 100048, China \\ ${ }^{4}$ Max-Planck-Institute for Mathematics in the Sciences, 04103, Leipzig, Germany
}

\begin{abstract}
Quantum entanglement plays essential roles in quantum information processing. The monogamy and polygamy relations characterize the entanglement distributions in the multipartite systems. We present a class of monogamy inequalities related to the $\mu$ th power of the entanglement measure based on Rényi- $\alpha$ entropy, as well as polygamy relations in terms of the $\mu$ th powered of Rényi- $\alpha$ entanglement of assistance. These monogamy and polygamy relations are shown to be tighter than the existing ones.
\end{abstract}

Keywords: monogamy relations, polygamy relations, Rényi- $\alpha$ entropy, Hamming weight

\section{INTRODUCTION}

Quantum entanglement is one of the most quintessential features of quantum mechanics, which distinguishes the quantum from the classical world and plays essential roles in quantum information processing [1 [5], revealing the basic understanding of the nature of quantum correlations. One distinct property of quantum entanglement is that a quantum system entangled with another system limits its sharing with other systems, known as the monogamy of entanglement [6, 7]. The monogamy of entanglement can be used as a resource to distribute a secret key which is secure against unauthorized parties [8, 9]. It also plays a significant role in many field of physics such as foundations of quantum mechanics [10, 34], condensed matter physics [11], statistical mechanics [34], and even black-hole physics [12, 13].

The monogamy inequality was first introduced by Coffman-Kundu-Wootters(CKW), by using tangle as a bipartite entanglement measure in three-qubit systems [14], and then generalized to multiqubit systems based on various entanglement measure [15]. The assisted entanglement is a dual concept to bipartite entanglement measure, which shows polygamy relations in multiparty quantum systems. For a three-qubit state $\rho_{A B C}$, a polygamy inequality was introduced as [16] $\tau^{a}\left(\rho_{A \mid B C}\right) \leq \tau^{a}\left(\rho_{A \mid B}\right)+\tau^{a}\left(\rho_{A \mid C}\right)$, where $\tau^{a}\left(\rho_{A \mid B C}\right)=\max \sum_{i} p_{i} \tau\left(\left|\psi_{i}\right\rangle_{A \mid B}\right)$ is the tangle of assistance [16, 17], with the maximum taking over all possible pure state decompositions of $\rho_{A B}=\sum_{i} p_{i}\left|\psi_{i}\right\rangle_{A B}\left\langle\psi_{i}\right|$. This tangle-based polygamy inequality was extended to multiqubit systems and also high-dimensional quantum systems in terms of various entropy entanglement measures [18, 19]. General polygamy inequalities of entanglement is also established in arbitrary dimensional multipartite quantum systems [20 25].

In this paper, we investigate the monogamy and polygamy constraints based on the $\mu$ th power of entanglement measures in terms of the Rényi- $\alpha$ entropy for multiqubit systems. By using the Hamming weight of binary vectors we present a class of monogamy inequalities for multiqubit entanglement based on the $\mu$ th power of Rényi- $\alpha$ entanglement ( $\mathrm{R} \alpha \mathrm{E})$ [26] for $\mu \geq 1$. For $0 \leq \mu \leq 1$, we introduce a class of tight polygamy inequalities based on the $\mu$ th power of the Rényi- $\alpha$ entanglement of assistance ( $\mathrm{R} \alpha \mathrm{EoA})$. Then, we show that both the monogamy inequalities with $\mu \geq 1$ and the polygamy inequalities with $0 \leq \mu \leq 1$ can be further improved to be tighter under certain conditions. These monogamy and polygamy relations are shown to be tighter than the existing ones. Moreover, our monogamy inequality is shown to be more effective for the counterexamples of the CKW monogamy inequality in higher-dimensional systems.

\section{PRELIMINARIES}

We first recall the conceptions of Rényi- $\alpha$ entropy, Rényi- $\alpha$ entanglement, and multiqubit monogamy and polygamy inequalities. For any $\alpha>0, \alpha \neq 1$, the Rényi- $\alpha$ entropy of a quantum state $\rho$ is defined as [27]

$$
S_{\alpha}(\rho)=\frac{1}{1-\alpha} \log \left(\operatorname{tr} \rho^{\alpha}\right) .
$$

*Electronic address: libobeijing2008@163.com. 
$S_{\alpha}(\rho)$ reduces to the von Neumann entropy when $\alpha$ approach to 1.

The Rényi- $\alpha$ entanglement $(\mathrm{R} \alpha \mathrm{E}) E_{\alpha}\left(|\psi\rangle_{A B}\right)$ of a bipartite pure state $|\psi\rangle_{A B}$ is defined as

$$
E_{\alpha}\left(|\psi\rangle_{A B}\right)=S_{\alpha}\left(\rho_{A}\right),
$$

where $\rho_{A}=\operatorname{Tr}_{B}|\psi\rangle_{A B}\langle\psi|$ is the reduced state of system $A$. For a mixed state $\rho_{A B}$, the Rényi- $\alpha$ entanglement is given by

$$
E_{\alpha}\left(\rho_{A \mid B}\right)=\min \sum_{i} p_{i} E_{\alpha}\left(\left|\psi_{i}\right\rangle_{A \mid B}\right)
$$

where the minimum is taken over all possible pure state decompositions of $\rho_{A B}=\sum_{i} p_{i}\left|\psi_{i}\right\rangle_{A B}\left\langle\psi_{i}\right|$.

As a dual concept to $\mathrm{R} \alpha \mathrm{E}$, the Rényi- $\alpha$ entanglement of assistance ( $\mathrm{R} \alpha \mathrm{EoA})$ is introduced as

$$
E_{\alpha}^{a}\left(\rho_{A \mid B}\right)=\max \sum_{i} p_{i} E_{\alpha}\left(\left|\psi_{i}\right\rangle_{A \mid B}\right),
$$

where the maximum is taken over all possible pure state decompositions of $\rho_{A B}[28]$.

For any multiqubit state $\rho_{A B_{0} \cdots B_{N-1}}$, a monogamous inequality has been presented in Ref. [28] for $\alpha \geq 2$,

$$
E_{\alpha}\left(\rho_{A \mid B_{0} \cdots B_{N-1}}\right) \geq \sum_{i=0}^{N-1} E_{\alpha}\left(\rho_{A \mid B_{i}}\right),
$$

where $E_{\alpha}\left(\rho_{A \mid B_{0} \cdots B_{N-1}}\right)$ is the $\mathrm{R} \alpha \mathrm{E}$ of $\rho_{A B_{0} \cdots B_{N}-1}$ with respect to the bipartition between $A$ and $B_{0} \cdots B_{N-1}$, and $E_{\alpha}\left(\rho_{A \mid B_{i}}\right)$ is the $\mathrm{R} \alpha \mathrm{E}$ of the reduced density matrix $\rho_{A B_{i}}, i=0, \cdots, N-1$.

In addition, a class of polygamy inequalities has been obtained for multiqubit systems,

$$
E_{\alpha}^{a}\left(\rho_{A \mid B_{0} \cdots B_{N-1}}\right) \leq \sum_{i=0}^{N-1} E_{\alpha}^{a}\left(\rho_{A \mid B_{i}}\right),
$$

for $0 \leq \alpha \leq 2, \alpha \neq 1$, where $E_{\alpha}\left(\rho_{A B_{0} \cdots B_{N-1}}\right)$ is the $\mathrm{R} \alpha \mathrm{EoA}$ of $\rho_{A B_{0} \cdots B_{N}-1}$ with respect to the bipartition between $A$ and $B_{0} \cdots B_{N-1}$, and $E_{\alpha}^{a}\left(\rho_{A \mid B_{i}}\right)$ is the $\mathrm{R} \alpha \mathrm{EoA}$ of the reduced density matrix $\rho_{A B_{i}}, i=0, \cdots, N-1$.

In Ref. [29], Kim established a class of tight monogamy inequalities of multiqubit entanglement in terms of Hamming weight. For any nonnegative integer $j$ with binary expansion $j=\sum_{i=0}^{n-1} j_{i} 2^{i}$, where $\log _{2} j \leq n$ and $j_{i} \in\{0,1\}$ for $i=0, \cdots, n-1$, one can always define a unique binary vector associated with $j, \vec{j}=\left(j_{0}, j_{1}, \cdots, j_{n-1}\right)$. The Hamming weight $\omega_{H}(\vec{j})$ of the binary vector $\vec{j}$ is defined to be the number of $1^{\prime} s$ in its coordinates [35]. Moreover, the Hamming weight $\omega_{H}(\vec{j})$ is bounded above by $\log _{2} j$,

$$
\omega_{H}(\vec{j}) \leq \log _{2} j \leq j
$$

Kim proposed the tight constraints of multiqubit entanglement based on Hamming weights [29],

$$
\left[E_{\alpha}\left(\rho_{\left.A \mid B_{0} B_{1} \ldots B_{N-1}\right)}\right)\right]^{\mu} \geq \sum_{j=0}^{N-1} \mu^{\omega_{H}(\vec{j})}\left[E_{\alpha}\left(\rho_{A \mid B_{j}}\right)\right]^{\mu}
$$

for $\mu \geq 1$, and

$$
\left[E_{\alpha}^{a}\left(\rho_{\left.A \mid B_{0} B_{1} \ldots B_{N-1}\right)}\right)\right]^{\mu} \leq \sum_{j=0}^{N-1} \mu^{\omega_{H}(\vec{\jmath})}\left[E_{\alpha}^{a}\left(\rho_{A \mid B_{j}}\right)\right]^{\mu}
$$

for $0 \leq \mu \leq 1$. Inequalities (5) and (6) are then further written as

$$
\left[E_{\alpha}\left(\rho_{A \mid B_{0} B_{1} \ldots B_{N-1}}\right)\right]^{\mu} \geq \sum_{j=0}^{N-1} \mu^{j}\left[E_{\alpha}\left(\rho_{A \mid B_{j}}\right)\right]^{\mu}
$$


for $\mu \geq 1$, and

$$
\left[E_{\alpha}^{a}\left(\rho_{A \mid B_{0} B_{1} \ldots B_{N-1}}\right)\right]^{\mu} \leq \sum_{j=0}^{N-1} \mu^{j}\left[E_{\alpha}^{a}\left(\rho_{A \mid B_{j}}\right)\right]^{\mu}
$$

for $0 \leq \mu \leq 1$.

In the following we show that these inequalities above can be further improved to be much tighter under certain conditions, which provide tighter constraints on the multiqubit entanglement distribution.

\section{TIGHTER CONSTRAINTS OF MULTIQUBIT ENTANGLEMENT IN TERMS OF R $\alpha$ E}

We first present a class of tighter monogamy and polygamy inequalities of multiqubit entanglement in terms of the $\mu$ th power of $\mathrm{R} \alpha \mathrm{E}$. We need the following results [32]. Suppose $k$ is a real number, $0<k \leq 1$. Then for any $0 \leq x \leq k$, we have

$$
(1+x)^{\mu} \geq 1+\frac{(1+k)^{\mu}-1}{k^{\mu}} x^{\mu}
$$

for $\mu \geq 1$, and

$$
(1+x)^{\mu} \leq 1+\frac{(1+k)^{\mu}-1}{k^{\mu}} x^{\mu}
$$

for $0 \leq \mu \leq 1$. Based on the inequality (7), we have the following theorem for $\mathrm{R} \alpha \mathrm{E}$.

Theorem 1 For any multiqubit state $\rho_{A B_{0} \ldots B_{N-1}}$ and $\alpha \geq 2$, we have

$$
\left[E_{\alpha}\left(\rho_{\left.A \mid B_{0} B_{1} \ldots B_{N-1}\right)}\right]^{\mu} \geq \sum_{j=0}^{N-1}\left(\frac{(1+k)^{\mu}-1}{k^{\mu}}\right)^{\omega_{H}(\vec{j})}\left[E_{\alpha}\left(\rho_{A \mid B_{j}}\right)\right]^{\mu},\right.
$$

where $\mu \geq 1, \vec{j}=\left(j_{0}, \cdots, j_{n-1}\right)$ is the vector from the binary representation of $j$, and $\omega_{H}(\vec{j})$ is the Hamming weight of $\vec{j}$.

[Proof] We first prove that

$$
\left[\sum_{j=0}^{N-1} E_{\alpha}\left(\rho_{A \mid B_{j}}\right)\right]^{\mu} \geq \sum_{j=0}^{N-1}\left(\frac{(1+k)^{\mu}-1}{k^{\mu}}\right)^{\omega_{H}(\vec{j})}\left[E_{\alpha}\left(\rho_{A \mid B_{j}}\right)\right]^{\mu} .
$$

Without loss of generality, we assume that the qubit subsystems $B_{0}, \ldots, B_{N-1}$ are so labeled such that

$$
k E_{\alpha}\left(\rho_{A \mid B_{j}}\right) \geq E_{\alpha}\left(\rho_{A \mid B_{j+1}}\right) \geq 0
$$

for $j=0,1, \ldots, N-2$ and some $0<k \leq 1$.

We first show that the inequality (10) holds for the case of $N=2^{n}$. For $n=1$, let $\rho_{A B_{0}}$ and $\rho_{A B_{1}}$ be the two-qubit reduced density matrices of a three-qubit pure state $\rho_{A B_{0} B_{1}}$. We obtain

$$
\left[E_{\alpha}\left(\rho_{A \mid B_{0}}\right)+E_{\alpha}\left(\rho_{A \mid B_{1}}\right)\right]^{\mu}=\left[E_{\alpha}\left(\rho_{A \mid B_{0}}\right)\right]^{\mu}\left(1+\frac{E_{\alpha}\left(\rho_{A \mid B_{1}}\right)}{E_{\alpha}\left(\rho_{A \mid B_{0}}\right)}\right)^{\mu} .
$$

Combining (7) and (11), we have

$$
\left(1+\frac{E_{\alpha}\left(\rho_{A \mid B_{1}}\right)}{E_{\alpha}\left(\rho_{A \mid B_{0}}\right)}\right)^{\mu} \geq 1+\frac{(1+k)^{\mu}-1}{k^{\mu}}\left(\frac{E_{\alpha}\left(\rho_{A \mid B_{1}}\right)}{E_{\alpha}\left(\rho_{A \mid B_{0}}\right)}\right)^{\mu} .
$$

From (12) and (13), we get

$$
\left[E_{\alpha}\left(\rho_{A \mid B_{0}}\right)+E_{\alpha}\left(\rho_{A \mid B_{1}}\right)\right]^{\mu} \geq\left[E_{\alpha}\left(\rho_{A \mid B_{0}}\right)\right]^{\mu}+\frac{(1+k)^{\mu}-1}{k^{\mu}}\left[E_{\alpha}\left(\rho_{A \mid B_{1}}\right)\right]^{\mu} .
$$


Therefore, the inequality (10) holds for $n=1$.

We assume that the inequality (10) holds for $N=2^{n-1}$ with $n \geq 2$, and prove the case of $N=2^{n}$. For an $(N+1)$-qubit pure state $\rho_{A B_{0} B_{1} \ldots B_{N-1}}$, we have $E_{\alpha}\left(\rho_{A \mid B_{j+2^{n-1}}}\right) \leq k^{2^{n-1}} E_{\alpha}\left(\rho_{A \mid B_{j}}\right)$ from (11). Therefore,

$$
0 \leq \frac{\sum_{j=2^{n-1}}^{2^{n}-1} E_{\alpha}\left(\rho_{A \mid B_{j}}\right)}{\sum_{j=0}^{2^{n-1}-1} E_{\alpha}\left(\rho_{A \mid B_{j}}\right)} \leq k^{2^{n-1}} \leq k
$$

and

$$
\left(\sum_{j=0}^{N-1} E_{\alpha}\left(\rho_{A \mid B_{j}}\right)\right)^{\mu}=\left(\sum_{j=0}^{2^{n-1}-1} E_{\alpha}\left(\rho_{A \mid B_{j}}\right)\right)^{\mu}\left(1+\frac{\sum_{j=2^{n-1}}^{2^{n}-1} E_{\alpha}\left(\rho_{A \mid B_{j}}\right)}{\sum_{j=0}^{2^{n-1}-1} E_{\alpha}\left(\rho_{A \mid B_{j}}\right)}\right)^{\mu} .
$$

Thus, we have

$$
\left(\sum_{j=0}^{N-1} E_{\alpha}\left(\rho_{A \mid B_{j}}\right)\right)^{\mu} \geq\left(\sum_{j=0}^{2^{n-1}-1} E_{\alpha}\left(\rho_{A \mid B_{j}}\right)\right)^{\mu}+\frac{(1+k)^{\mu}-1}{k^{\mu}}\left(\sum_{j=2^{n-1}}^{2^{n}-1} E_{\alpha}\left(\rho_{A \mid B_{j}}\right)\right)^{\mu} .
$$

According to the induction hypothesis, we get

$$
\left(\sum_{j=0}^{2^{n-1}-1} E_{\alpha}\left(\rho_{A \mid B_{j}}\right)\right)^{\mu} \geq \sum_{j=0}^{2^{n-1}-1}\left(\frac{(1+k)^{\mu}-1}{k^{\mu}}\right)^{\omega_{H}(\vec{j})}\left[E_{\alpha}\left(\rho_{A \mid B_{j}}\right)\right]^{\mu} .
$$

By relabeling the subsystems, the induction hypothesis leads to

$$
\left(\sum_{j=2^{n-1}}^{2^{n}-1} E_{\alpha}\left(\rho_{A \mid B_{j}}\right)\right)^{\mu} \geq \sum_{j=2^{n-1}}^{2^{n}-1}\left(\frac{(1+k)^{\mu}-1}{k^{\mu}}\right)^{\omega_{H}(\vec{j})-1}\left[E_{\alpha}\left(\rho_{A \mid B_{j}}\right)\right]^{\mu} .
$$

Thus, we have

$$
\left(\sum_{j=0}^{2^{n}-1} E_{\alpha}\left(\rho_{A \mid B_{j}}\right)\right)^{\mu} \geq \sum_{j=0}^{2^{n}-1}\left(\frac{(1+k)^{\mu}-1}{k^{\mu}}\right)^{\omega_{H}(\vec{j})}\left[E_{\alpha}\left(\rho_{A \mid B_{j}}\right)\right]^{\mu} .
$$

Now consider a $\left(2^{n}+1\right)$-qubit state

$$
\Gamma_{A B_{0} B_{1} \ldots B_{2^{n}-1}}=\rho_{A B_{0} B_{1} \ldots B_{N-1}} \otimes \sigma_{B_{N} \ldots B_{2^{n}-1}},
$$

which is the tensor product of $\rho_{A B_{0} B_{1} \ldots B_{N-1}}$ and an arbitrary $\left(2^{n}-N\right)$-qubit state $\sigma_{B_{N} \ldots B_{2^{n}-1}}$. We have

$$
\left[E_{\alpha}\left(\Gamma_{A \mid B_{0} B_{1} \ldots B_{2^{n}-1}}\right)\right]^{\mu} \geq \sum_{j=0}^{2^{n}-1}\left(\frac{(1+k)^{\mu}-1}{k^{\mu}}\right)^{\omega_{H}(\vec{j})}\left[E_{\alpha}\left(\Gamma_{A \mid B_{j}}\right)\right]^{\mu},
$$

where $\Gamma_{A \mid B_{j}}$ is the two-qubit reduced density matrix of $\Gamma_{A B_{0} B_{1} \ldots B_{2^{n}-1}}, j=0,1, \ldots, 2^{n}-1$. Therefore,

$$
\begin{aligned}
{\left[E _ { \alpha } \left(\rho_{\left.\left.A \mid B_{0} B_{1} \ldots B_{N-1}\right)\right]^{\mu}}\right.\right.} & =\left[E _ { \alpha } \left(\Gamma_{\left.\left.A \mid B_{0} B_{1} \ldots B_{2^{n}-1}\right)\right]^{\mu}}\right.\right. \\
& \geq \sum_{j=0}^{2^{n}-1}\left(\frac{(1+k)^{\mu}-1}{k^{\mu}}\right)^{\omega_{H}(\vec{j})}\left[E_{\alpha}\left(\Gamma_{A \mid B_{j}}\right)\right]^{\mu} \\
& =\sum_{j=0}^{N-1}\left(\frac{(1+k)^{\mu}-1}{k^{\mu}}\right)^{\omega_{H}(\vec{j})}\left[E_{\alpha}\left(\rho_{A \mid B_{j}}\right)\right]^{\mu},
\end{aligned}
$$

where $\Gamma_{A \mid B_{0} B_{1} \ldots B_{2^{n}-1}}$ is separated to the bipartition $A B_{0} \ldots B_{N-1}$ and $B_{N} \ldots B_{2^{n}-1}, E_{\alpha}\left(\Gamma_{A \mid B_{0} B_{1} \cdots B_{2^{n}-1}}\right)=$ $E_{\alpha}\left(\rho_{A \mid B_{0} B_{1} \cdots B_{N-1}}\right), E_{\alpha}\left(\Gamma_{A \mid B_{j}}\right)=0$ for $j=N, \cdots, 2^{n}-1$, and $\Gamma_{A B_{j}}=\rho_{A B_{j}}$ for each $j=0, \cdots, N-1$.

Since $\left(\frac{(1+k)^{\mu}-1}{k^{\mu}}\right)^{\omega_{H}(\vec{j})} \geq \mu^{\omega_{H}(\vec{j})}$ for $\mu \geq 1$, for any multiqubit state $\rho_{A B_{0} B_{1} \cdots B_{N-1}}$ we have the following relation,

$$
\left[E_{\alpha}\left(\rho_{A \mid B_{0} B_{1} \ldots B_{N-1}}\right)\right]^{\mu} \geq \sum_{j=0}^{N-1}\left(\frac{(1+k)^{\mu}-1}{k^{\mu}}\right)^{\omega_{H}(\vec{j})}\left[E_{\alpha}\left(\rho_{A \mid B_{j}}\right)\right]^{\mu} \geq \sum_{j=0}^{N-1} \mu^{\omega_{H}(\vec{j})}\left[E_{\alpha}\left(\rho_{A \mid B_{j}}\right)\right]^{\mu} .
$$




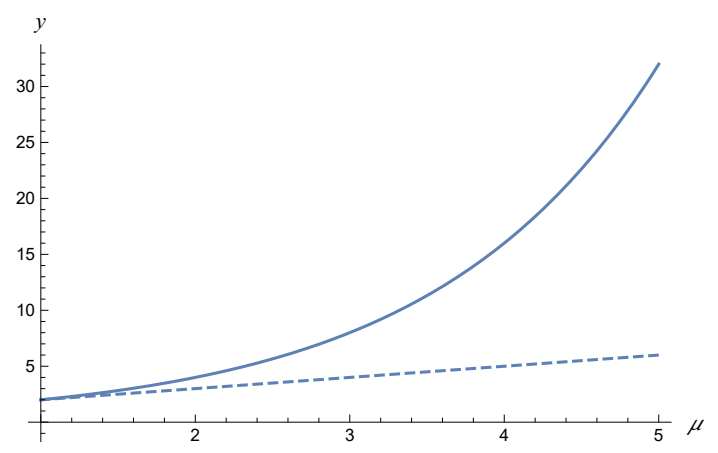

FIG. 1: Rényi- $\alpha$ entanglement with respect to $\mu$ : the solid line is for $y_{1}$ and the dashed line for $y_{2}$ from the result in 29].

Therefore, our inequality (9) in Theorem 1 is always tighter than the inequality (5).

In fact, the tighter monogamy inequality (9) holds not only for multiqubit systems, but also for some multipartite higher-dimensional quantum systems, which can be proved in a similar way as in [29]. Here, we show that (9) is also more efficient than (5) for such higher-dimensional quantum systems. Let us consider the counterexample of the CKW inequality in tripartite quantum systems [30],

$$
|\psi\rangle_{A B C}=\frac{1}{\sqrt{6}}(|123\rangle-|132\rangle+|231\rangle-|213\rangle+|312\rangle-|321\rangle) .
$$

One has $E_{\alpha}\left(|\psi\rangle_{A \mid B C}\right)=S_{\alpha}(\rho)$. Taking $\alpha=3$, we have $E_{\alpha}\left(|\psi\rangle_{A \mid B C}\right)=\log 3$ and the $\mathrm{R} \alpha \mathrm{E}$ of the two-qubit reduced density matrices are

$$
E_{\alpha}\left(\rho_{A \mid B}\right)=E_{\alpha}\left(\rho_{A \mid C}\right)=-\frac{1}{2} \log \operatorname{tr} \sigma_{A}^{3}=1
$$

In this case $k=1$, for $\mu \geq 1$, we have

$$
y_{1} \equiv\left[E_{\alpha}\left(\rho_{A \mid B}\right)\right]^{\mu}+\frac{(1+k)^{\mu}-1}{k^{\mu}}\left[E_{\alpha}\left(\rho_{A \mid C}\right)\right]^{\mu}=1+\frac{(1+k)^{\mu}-1}{k^{\mu}}=2^{\mu},
$$

and

$$
y_{2} \equiv\left[E_{\alpha}\left(\rho_{A \mid B}\right)\right]^{\mu}+\mu\left[E_{\alpha}\left(\rho_{A \mid C}\right)\right]^{\mu}=1+\mu .
$$

Therefore, one gets

$$
\left[E_{\alpha}\left(\rho_{A \mid B}\right)\right]^{\mu}+\frac{(1+k)^{\mu}-1}{k^{\mu}}\left[E_{\alpha}\left(\rho_{A \mid C}\right)\right]^{\mu} \geq\left[E_{\alpha}\left(\rho_{A \mid B}\right)\right]^{\mu}+\mu\left[E_{\alpha}\left(\rho_{A \mid C}\right)\right]^{\mu} .
$$

where $\mu \geq 1$, see Fig. 1 . In other words, our new monogamy inequality is indeed tighter than the previous one given in [29].

Under certain conditions, the inequality (9) can even be improved further to become a much tighter inequality.

Theorem 2 For $\mu \geq 1, \alpha \geq 2$ and real number $0<k \leq 1$, any multiqubit state $\rho_{A B_{0} \ldots B_{N-1}}$ satisfies

$$
\left[E_{\alpha}\left(\rho_{A \mid B_{0} \ldots B_{N-1}}\right)\right]^{\mu} \geq \sum_{j=0}^{N-1}\left(\frac{(1+k)^{\mu}-1}{k^{\mu}}\right)^{j}\left[E_{\alpha}\left(\rho_{A \mid B_{j}}\right)\right]^{\mu},
$$

if

$$
k E_{\alpha}\left(\rho_{A \mid B_{j}}\right) \geq \sum_{j=i+1}^{N-1} E_{\alpha}\left(\rho_{A \mid B_{j}}\right)
$$

for $i=0,1, \ldots, N-2$.

[Proof] We need to show

$$
\left(\sum_{j=0}^{N-1} E_{\alpha}\left(\rho_{A \mid B_{j}}\right)\right)^{\mu} \geq \sum_{j=0}^{N-1}\left(\frac{(1+k)^{\mu}-1}{k^{\mu}}\right)^{j}\left(E_{\alpha}\left(\rho_{A \mid B_{j}}\right)\right)^{\mu} .
$$


For any multiqubit state $\rho_{A B_{0} \ldots B_{N-1}}$, it is easy to show that

$$
\left(\sum_{j=0}^{N-1} E_{\alpha}\left(\rho_{A \mid B_{j}}\right)\right)^{\mu}=\left(E_{\alpha}\left(\rho_{A \mid B_{0}}\right)\right)^{\mu}\left(1+\frac{\sum_{j=1}^{N-1} E_{\alpha}\left(\rho_{A \mid B_{j}}\right)}{E_{\alpha}\left(\rho_{A \mid B_{0}}\right)}\right)^{\mu}
$$

and

$$
\left(1+\frac{\sum_{j=1}^{N-1} E_{\alpha}\left(\rho_{A \mid B_{j}}\right)}{E_{\alpha}\left(\rho_{A \mid B_{0}}\right)}\right)^{\mu} \geq 1+\left(\frac{(1+k)^{\mu}-1}{k^{\mu}}\right)\left(\frac{\sum_{j=1}^{N-1} E_{\alpha}\left(\rho_{A \mid B_{j}}\right)}{E_{\alpha}\left(\rho_{A \mid B_{0}}\right)}\right)^{\mu} .
$$

Thus,

$$
\begin{aligned}
\left(\sum_{j=0}^{N-1} E_{\alpha}\left(\rho_{A \mid B_{j}}\right)\right)^{\mu} & \geq\left(E_{\alpha}\left(\rho_{A \mid B_{0}}\right)\right)^{\mu}+\left(\frac{(1+k)^{\mu}-1}{k^{\mu}}\right)\left(\sum_{j=1}^{N-1} E_{\alpha}\left(\rho_{A \mid B_{j}}\right)\right)^{\mu} \\
& \geq \sum_{j=0}^{N-1}\left(\frac{(1+k)^{\mu}-1}{k^{\mu}}\right)^{j}\left(E_{\alpha}\left(\rho_{A \mid B_{j}}\right)\right)^{\mu}
\end{aligned}
$$

where the second inequality is due to the induction hypothesis.

In fact, according to (4), for any $\mu \geq 1$, one has

$$
\begin{aligned}
{\left[E_{\alpha}\left(\rho_{A \mid B_{0} \ldots B_{N-1}}\right)\right]^{\mu} } & \geq \sum_{j=0}^{N-1}\left(\frac{(1+k)^{\mu}-1}{k^{\mu}}\right)^{j}\left(E_{\alpha}\left(\rho_{A \mid B_{j}}\right)\right)^{\mu} \\
& \geq \sum_{j=0}^{N-1}\left(\frac{(1+k)^{\mu}-1}{k^{\mu}}\right)^{\omega_{H}(\vec{j})}\left[E_{\alpha}\left(\rho_{A \mid B_{j}}\right)\right]^{\mu} .
\end{aligned}
$$

For the case of $\mu<0$, we can also derive a tighter upper bound of $E_{\alpha}^{\mu}\left(\rho_{A \mid B_{0} B_{1} \ldots B_{N-1}}\right)$.

Theorem 3 For any multiqubit state $\rho_{A B_{0} \ldots B_{N-1}}$ with $E_{\alpha}\left(\rho_{A B_{i}}\right) \neq 0, i=0,1, \ldots, N-1$, we have

$$
\left[E_{\alpha}\left(\rho_{A \mid B_{0} B_{1} \ldots B_{N-1}}\right)\right]^{\mu} \leq \frac{1}{N-1} \sum_{j=0}^{N-1}\left[E_{\alpha}\left(\rho_{A \mid B_{j}}\right)\right]^{\mu},
$$

for all $\mu<0$ and $\alpha \geq 2$.

[Proof] Similar to the proof in [31], for arbitrary three-qubit states we have

$$
\begin{aligned}
{\left[E_{\alpha}\left(\rho_{A \mid B_{0} B_{1}}\right)\right]^{\mu} } & \leq\left[E_{\alpha}^{2}\left(\rho_{A \mid B_{0}}\right)+E_{\alpha}^{2}\left(\rho_{A \mid B_{1}}\right)\right]^{\frac{\mu}{2}} \\
& =\left[E_{\alpha}\left(\rho_{A \mid B_{0}}\right)\right]^{\mu}\left(1+\frac{E_{\alpha}^{2}\left(\rho_{A \mid B_{1}}\right)}{E_{\alpha}^{2}\left(\rho_{A \mid B_{0}}\right)}\right)^{\frac{\mu}{2}} \\
& <\left[E_{\alpha}\left(\rho_{A \mid B_{0}}\right)\right]^{\mu}
\end{aligned}
$$

where the first inequality is from $\mu<0$, the second inequality is due to $\left(1+\frac{E_{\alpha}^{2}\left(\rho_{A \mid B_{1}}\right)}{E_{\alpha}^{2}\left(\rho_{A \mid B_{0}}\right)}\right)^{\mu}<1$. Moreover, we have

$$
\left[E_{\alpha}\left(\rho_{A \mid B_{0} B_{1}}\right)\right]^{\mu}<\left[E_{\alpha}\left(\rho_{A \mid B_{1}}\right)\right]^{\mu} .
$$

Combining (20) and (21), we get

$$
\left[E_{\alpha}\left(\rho_{A \mid B_{0} B_{1}}\right)\right]^{\mu}<\frac{1}{2}\left\{\left[E_{\alpha}\left(\rho_{A \mid B_{0}}\right)\right]^{\mu}+\left[E_{\alpha}\left(\rho_{A \mid B_{1}}\right)\right]^{\mu}\right\} .
$$

Thus, we obtain

$$
\begin{aligned}
& {\left[E_{\alpha}\left(\rho_{\left.A \mid B_{0} B_{1} \ldots B_{N-1}\right)}\right)\right]^{\mu} } \\
< & \frac{1}{2}\left\{\left[E_{\alpha}\left(\rho_{A \mid B_{0}}\right)\right]^{\mu}+\left[E_{\alpha}\left(\rho_{A \mid B_{1} \ldots B_{N-1}}\right)\right]^{\mu}\right\} \\
< & \frac{1}{2}\left[E_{\alpha}\left(\rho_{A \mid B_{0}}\right)\right]^{\mu}+\left(\frac{1}{2}\right)^{2}\left[E_{\alpha}\left(\rho_{A \mid B_{1}}\right)\right]^{\mu}+\left(\frac{1}{2}\right)^{2}\left[E_{\alpha}\left(\rho_{A \mid B_{2} \ldots B_{N-1}}\right)\right]^{\mu} \\
< & \ldots \\
< & \frac{1}{2}\left[E_{\alpha}\left(\rho_{A \mid B_{0}}\right)\right]^{\mu}+\left(\frac{1}{2}\right)^{2}\left[E_{\alpha}\left(\rho_{A \mid B_{1}}\right)\right]^{\mu}+\ldots+\left(\frac{1}{2}\right)^{N-2}\left[E_{\alpha}\left(\rho_{A \mid B_{N-2}}\right)\right]^{\mu}+\left(\frac{1}{2}\right)^{N-2}\left[E_{\alpha}\left(\rho_{A \mid B_{N-1}}\right)\right]^{\mu} .
\end{aligned}
$$


One can get a set of inequalities through the cyclic permutation of the pair indices $B_{0}, B_{1}, \ldots, B_{N-1}$ in (22). Summing up these inequalities, we get (19).

\section{TIGHTER CONSTRAINTS OF MULTIQUBIT ENTANGLEMENT IN TERMS OF R $\alpha$ EA}

We consider now the Rényi- $\alpha$ entanglement of assistance (R $\alpha$ EoA) defined in (1), and provide a class of polygamy inequalities satisfied by the multiqubit entanglement in terms of R $\alpha$ EoA.

Theorem 4 For any multiqubit state $\rho_{A B_{0} \ldots B_{N-1}}$ and $0 \leq \mu \leq 1,0<\alpha<2, \alpha \neq 1$, we have

$$
\left[E_{\alpha}^{a}\left(\rho_{A \mid B_{0} B_{1} \ldots B_{N-1}}\right)\right]^{\mu} \leq \sum_{j=0}^{N-1}\left(\frac{(1+k)^{\mu}-1}{k^{\mu}}\right)^{\omega_{H}(\vec{j})}\left[E_{\alpha}^{a}\left(\rho_{A \mid B_{j}}\right)\right]^{\mu} .
$$

[Proof] Similar to proof in Ref. [29], we just need to prove

$$
\left[\sum_{j=0}^{N-1} E_{\alpha}^{a}\left(\rho_{A \mid B_{j}}\right)\right]^{\mu} \leq \sum_{j=0}^{N-1}\left(\frac{(1+k)^{\mu}-1}{k^{\mu}}\right)^{\omega_{H}(\vec{j})}\left[E_{\alpha}^{a}\left(\rho_{A \mid B_{j}}\right)\right]^{\mu}
$$

Firstly, assume that the qubit subsystems $B_{0}, \ldots, B_{N-1}$ satisfies

$$
k E_{\alpha}^{a}\left(\rho_{A \mid B_{j}}\right) \geq E_{\alpha}^{a}\left(\rho_{A \mid B_{j+1}}\right) \geq 0,
$$

where $j=0,1, \ldots, N-2$ and $0<k \leq 1$. Similar to the proof of Theorem 1 , we first show that the inequality (24) holds for a three-qubit pure state $\rho_{A B_{0} B_{1}}$. We have

$$
\begin{aligned}
{\left[E_{\alpha}^{a}\left(\rho_{A \mid B_{0}}\right)+E_{\alpha}^{a}\left(\rho_{A \mid B_{1}}\right)\right]^{\mu} } & =\left[E_{\alpha}^{a}\left(\rho_{A \mid B_{0}}\right)\right]^{\mu}\left(1+\frac{E_{\alpha}^{a}\left(\rho_{A \mid B_{1}}\right)}{E_{\alpha}^{a}\left(\rho_{A \mid B_{0}}\right)}\right)^{\mu} \\
& \leq\left[E_{\alpha}^{a}\left(\rho_{A \mid B_{0}}\right)\right]^{\mu}\left[1+\frac{(1+k)^{\mu}-1}{k^{\mu}}\left(\frac{E_{\alpha}^{a}\left(\rho_{A \mid B_{1}}\right)}{E_{\alpha}^{a}\left(\rho_{A \mid B_{0}}\right)}\right)^{\mu}\right] \\
& =\left[E_{\alpha}^{a}\left(\rho_{A \mid B_{0}}\right)\right]^{\mu}+\frac{(1+k)^{\mu}-1}{k^{\mu}}\left[E_{\alpha}^{a}\left(\rho_{A \mid B_{1}}\right)\right]^{\mu}
\end{aligned}
$$

where the inequality is due to (8).

Then we assume that the inequality (24) holds for $N=2^{n-1}$ with $n \geq 2$. Consider the case of $N=2^{n}$. For an $(N+1)$-qubit pure state $\rho_{A B_{0} B_{1} \ldots B_{N-1}}$ with its two-qubit reduced density matrices $\rho_{A B_{j}}, j=0,1, \ldots, N-1$, we have $E_{\alpha}^{a}\left(\rho_{A \mid B_{j+2^{n-1}}}\right) \leq k^{2^{n-1}} E_{\alpha}^{a}\left(\rho_{A \mid B_{j}}\right)$ due to the ordering of subsystems in the inequality (25). Then, we get

$$
0 \leq \frac{\sum_{j=2^{n-1}}^{2^{n}-1} E_{\alpha}^{a}\left(\rho_{A \mid B_{j}}\right)}{\sum_{j=0}^{2^{n-1}-1} E_{\alpha}^{a}\left(\rho_{A \mid B_{j}}\right)} \leq k^{2^{n-1}} \leq k \leq 1
$$

and

$$
\left(\sum_{j=0}^{N-1} E_{\alpha}^{a}\left(\rho_{A \mid B_{j}}\right)\right)^{\mu}=\left(\sum_{j=0}^{2^{n-1}-1} E_{\alpha}^{a}\left(\rho_{A \mid B_{j}}\right)\right)^{\mu}\left(1+\frac{\sum_{j=2^{n-1}}^{2^{n}-1} E_{\alpha}^{a}\left(\rho_{A \mid B_{j}}\right)}{\sum_{j=0}^{2^{n-1}-1} E_{\alpha}^{a}\left(\rho_{A \mid B_{j}}\right)}\right)^{\mu}
$$

Hence,

$$
\left(\sum_{j=0}^{N-1} E_{\alpha}^{a}\left(\rho_{A \mid B_{j}}\right)\right)^{\mu} \leq\left(\sum_{j=0}^{2^{n-1}-1} E_{\alpha}\left(\rho_{A \mid B_{j}}\right)\right)^{\mu}+\frac{(1+k)^{\mu}-1}{k^{\mu}}\left(\sum_{j=2^{n-1}}^{2^{n}-1} E_{\alpha}^{a}\left(\rho_{A \mid B_{j}}\right)\right)^{\mu}
$$

According to the induction hypothesis, we get

$$
\left(\sum_{j=0}^{2^{n-1}-1} E_{\alpha}^{a}\left(\rho_{A \mid B_{j}}\right)\right)^{\mu} \leq \sum_{j=0}^{2^{n-1}-1}\left(\frac{(1+k)^{\mu}-1}{k^{\mu}}\right)^{\omega_{H}(\vec{j})}\left[E_{\alpha}^{a}\left(\rho_{A \mid B_{j}}\right)\right]^{\mu} .
$$


By relabeling the subsystems, the induction hypothesis leads to

$$
\left(\sum_{j=2^{n-1}}^{2^{n}-1} E_{\alpha}^{a}\left(\rho_{A \mid B_{j}}\right)\right)^{\mu} \leq \sum_{j=2^{n-1}}^{2^{n}-1}\left(\frac{(1+k)^{\mu}-1}{k^{\mu}}\right)^{\omega_{H}(\vec{j})-1}\left[E_{\alpha}^{a}\left(\rho_{A \mid B_{j}}\right)\right]^{\mu} .
$$

Therefore,

$$
\left(\sum_{j=0}^{2^{n}-1} E_{\alpha}^{a}\left(\rho_{A \mid B_{j}}\right)\right)^{\mu} \leq \sum_{j=0}^{2^{n}-1}\left(\frac{(1+k)^{\mu}-1}{k^{\mu}}\right)^{\omega_{H}(\vec{j})}\left[E_{\alpha}^{a}\left(\rho_{A \mid B_{j}}\right)\right]^{\mu} .
$$

Consider the $\left(2^{n}+1\right)$-qubit state (14). We have

$$
\begin{aligned}
{\left[E_{\alpha}^{a}\left(\rho_{\left.A \mid B_{0} B_{1} \ldots B_{N-1}\right)}\right)\right]^{\mu} } & =\left[E_{\alpha}^{a}\left(\Gamma_{A \mid B_{0} B_{1} \ldots B_{2^{n}-1}}\right)\right]^{\mu} \\
& \leq \sum_{j=0}^{2^{n}-1}\left(\frac{(1+k)^{\mu}-1}{k^{\mu}}\right)^{\omega_{H}(\vec{j})}\left[E_{\alpha}^{a}\left(\Gamma_{A \mid B_{j}}\right)\right]^{\mu} \\
& =\sum_{j=0}^{N-1}\left(\frac{(1+k)^{\mu}-1}{k^{\mu}}\right)^{\omega_{H}(\vec{j})}\left[E_{\alpha}^{a}\left(\rho_{A \mid B_{j}}\right)\right]^{\mu} .
\end{aligned}
$$

Since $\frac{(1+k)^{\mu}-1}{k^{\mu}} \leq \mu$ for $0 \leq \mu \leq 1$, it is easy to see that (23) is tighter than (6).

As an example, let us consider the three-qubit W-state [33],

$$
|W\rangle_{A B C}=\frac{1}{\sqrt{3}}(|211\rangle+|121\rangle+|112\rangle) .
$$

We have $E_{\alpha}^{a}\left(|\psi\rangle_{A \mid B C}\right)=S_{\alpha}(\rho)=\log 3-\frac{2}{3}$ and

$$
E_{\alpha}^{a}\left(\rho_{A \mid B}\right)=E_{\alpha}^{a}\left(\rho_{A \mid C}\right)=-\frac{1}{2} \log \operatorname{tr} \sigma_{A}^{3}=\frac{2}{3} .
$$

In the case of $k=1$ and $0 \leq \mu \leq 1$, we have

$$
y_{3} \equiv\left[E_{\alpha}^{a}\left(\rho_{A \mid B}\right)\right]^{\mu}+\frac{(1+k)^{\mu}-1}{k^{\mu}}\left[E_{\alpha}^{a}\left(\rho_{A \mid C}\right)\right]^{\mu}=\left(\frac{2}{3}\right)^{\mu}+\frac{(1+k)^{\mu}-1}{k^{\mu}}\left(\frac{2}{3}\right)^{\mu}=\left(\frac{2}{3}\right)^{\mu} 2^{\mu},
$$

and

$$
y_{4} \equiv\left[E_{\alpha}^{a}\left(\rho_{A \mid B}\right)\right]^{\mu}+\mu\left[E_{\alpha}^{a}\left(\rho_{A \mid C}\right)\right]^{\mu}=\left(\frac{2}{3}\right)^{\mu}(1+\mu) .
$$

Therefore, we get

$$
\left[E_{\alpha}^{a}\left(\rho_{A \mid B}\right)\right]^{\mu}+\frac{(1+k)^{\mu}-1}{k^{\mu}}\left[E_{\alpha}^{a}\left(\rho_{A \mid C}\right)\right]^{\mu} \leq\left[E_{\alpha}\left(\rho_{A \mid B}\right)\right]^{\mu}+\mu\left[E_{\alpha}\left(\rho_{A \mid C}\right)\right]^{\mu}
$$

where $0 \leq \mu \leq 1$, see Fig. 2 .

Similar to the improvement from the inequality (9) to the inequality (16), we can also improve the polygamy inequality in Theorem 4 . The proof is similar to the Theorem 2 .

Theorem 5 For $0 \leq \mu \leq 1,0<\alpha<2, \alpha \neq 1$ and $0<k \leq 1$, we have for any multiqubit state $\rho_{A B_{0} \ldots B_{N-1}}$,

$$
\left[E_{\alpha}^{a}\left(\rho_{A \mid B_{0} \ldots B_{N-1}}\right)\right]^{\mu} \leq \sum_{j=0}^{N-1}\left(\frac{(1+k)^{\mu}-1}{k^{\mu}}\right)^{j}\left[E_{\alpha}^{a}\left(\rho_{A \mid B_{j}}\right)\right]^{\mu},
$$

if

$$
k E_{\alpha}^{a}\left(\rho_{A \mid B_{i}}\right) \geq \sum_{j=i+1}^{N-1} E_{\alpha}^{a}\left(\rho_{A \mid B_{j}}\right)
$$

for $i=0,1, \ldots, N-2$.

Since $\omega_{H}(\vec{j}) \leq j$, for $0 \leq \mu \leq 1$ we obtain

$$
\begin{aligned}
{\left[E_{\alpha}^{a}\left(\rho_{A \mid B_{0} \ldots B_{N-1}}\right)\right]^{\mu} } & \leq \sum_{j=0}^{N-1}\left(\frac{(1+k)^{\mu}-1}{k^{\mu}}\right)^{j}\left(E_{\alpha}^{a}\left(\rho_{A \mid B_{j}}\right)\right)^{\mu} \\
& \leq \sum_{j=0}^{N-1}\left(\frac{(1+k)^{\mu}-1}{k^{\mu}}\right)^{\omega_{H}(\vec{j})}\left[E_{\alpha}^{a}\left(\rho_{A \mid B_{j}}\right)\right]^{\mu} .
\end{aligned}
$$

Therefore, for any multiqubit state $\rho_{A B_{0} \ldots B_{N-1}}$ satisfying the condition (28), the inequality (27) of Theorem 5 is tighter than the inequality (23) of Theorem 4 . 


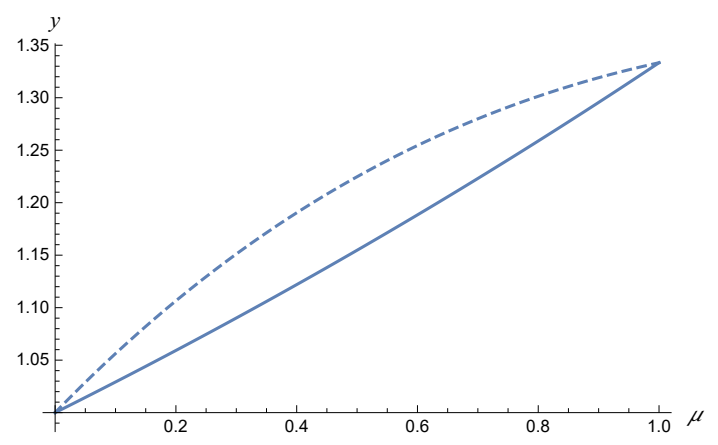

FIG. 2: Rényi- $\alpha$ entanglement $y$ with respect to $\mu$ : the solid line is for $y_{3}$ and the dashed line for $y_{4}$ from the result in [29].

\section{CONCLUSION}

Quantum entanglement is the essential resource in quantum information. The monogamy and polygamy relations characterize the entanglement distributions in the multipartite systems. Tighter monogamy and polygamy inequalities give finer characterization of the entanglement distribution. In this article, by using the Hamming weights of binary vectors we have proposed a class of monogamy inequalities related to the $\mu$ th power of the entanglement measure based on Rényi- $\alpha$ entropy, polygamy relations in terms of the $\mu$ th powered of of $\operatorname{R} \alpha$ EoA for $0 \leq \mu \leq 1$. These new monogamy and polygamy relations are shown to be tighter than the existing ones. Moreover, it has been shown that our monogamy inequality is effective for the counterexamples of the CKW monogamy inequality in higher-dimensional systems. Our results may highlight further investigations on the entanglement distribution in multipartite systems.

Acknowledgments This work is supported by NSFC under numbers 11765016, 11675113, NSF of Beijing under No. KZ201810028042, and Beijing Natural Science Foundation (Z190005).

[1] Mintert F, Kuś M and Buchleitner A 2004 Phys. Rev. Lett 92167902

[2] Chen K, Albeverio S and Fei S M 2005 Phys. Rev. Lett 95040504

[3] Breuer H P 2006 J. Phys. A: Math. Gen 3911847

[4] Zhao Y B, Zhang W L, Wang D, Song X T, Zhou L J and Ding C B 2019 Chin. Phys. B 28104203

[5] Li H M, Guo M D, Zhang R and Su X M 2019 Chin. Phys. B 28100302

[6] Terhal B M 2004 IBM J. Res. Dev. 4871

[7] Kim J S, Gour G and Sanders B C 2012 Contemp. Phys. 53417

[8] Renes J M and Grassl M 2006 Phys. Rev. A 74022317

[9] Masanes L 2009 Phys. Rev. Lett 102140501

[10] Toner B 2009 Proc. R. Soc. A 46559

[11] Garca-Saez A and Latorre J I 2013 Phys. Rev. B 87085130

[12] Susskind L 2013 arXiv: 1301.4505v2[hep-th]

[13] Lloyd S and Preskill J 2014 J. High Energy Phys 08126

[14] Coffman V, Kundu J and Wootters W K 2000 Phys. Rev. A 61052306 (2000)

[15] Osborne T J and Verstraete F 2006 Phys. Rev. Lett 96220503 (2006)

[16] Gour G, Bandyopadhay S and Sanders B C 2007 J. Math. Phys. 48012108 (2007)

[17] Gour G, Meyer D A and Sanders B C 2005 Phys. Rev. A 72042329 (2005)

[18] Kim J S 2010 Phys. Rev. A 81062328 (2010)

[19] Buscemi F, Gour G and Kim J S 2009 Phys. Rev. A 80012324

[20] Kim J S 2012 Phys. Rev. A 85062302

[21] Kim J S 2016 Phys. Rev. A 94062338

[22] Kim J S 2018 Phys. Rev. A 97012334

[23] Luo Y and Li Y 2015 Ann. Phys. (NY) 362511

[24] Zhu X N and Fei S M 2014 Phys. Rev. A 90024304

[25] Jin Z X, Li J, Li T and Fei S M 2018 Phys. Rev. A 97032336

[26] Horodecki R, Horodecki P and Horodecki M 1996 Phys. Lett. A 210377

[27] Kim J S, Sanders B C 2011 J. Phys. A: Math. Theor. 44295303

[28] Kim J S 2012 Phys. Rev. A 85032335

[29] Kim J S 2018 Sci. Rep. 812245 
[30] Ou Y 2007 Phys. Rev. A 75034305

[31] Jin Z X and Fei S M 2017 Quantum Inf. Process 1677

[32] Yang L M, Chen B, Fei S M and Wang Z X 2019 Commun. Theor. Phys. 71 545-554

[33] Kim J S 2018 Phys. Rev. A 97042332

[34] Bennett C H 2014 Proceedings of the FQXi 4th International Conference, January 5-10, 2014 Vieques Island, Puerto Rico

[35] Nielsen M A and Chuang I L 2000 Quantum Computation and Quantum Information (Cambridge, England: Canbrudge University Press) pp. 60-169 